\title{
The Reproducing Kernel Structure Arising from a Combination of Continuous and Discrete Orthogonal Polynomials into Fourier Systems
}

\author{
Luís Daniel Abreu \\ In memory of Joaquin Bustoz
}

\begin{abstract}
We study mapping properties of operators with kernels defined via a combination of continuous and discrete orthogonal polynomials, which provide an abstract formulation of quantum $(q$-) Fourier-type systems. We prove Ismail's conjecture regarding the existence of a reproducing kernel structure behind these kernels, by establishing a link with Saitoh's theory of linear transformations in Hilbert space. The results are illustrated with Fourier kernels with ultraspherical, their continuous $q$-extensions and generalizations. As a byproduct of this approach, a new class of sampling theorems is obtained, as well as Neumann-type expansions in Bessel and $q$-Bessel functions.
\end{abstract}

\section{Introduction}

The Gegenbauer expansion of the two variable complex exponential in terms of the ultraspherical polynomials

$$
e^{i x t}=\Gamma(v)\left(\frac{t}{2}\right)^{-v} \sum_{k=0}^{\infty} i^{k}(v+k) J_{v+k}(t) C_{k}^{v}(x)
$$

has the remarkable feature of being at the same time an expansion in a Neumann series of Bessel functions. The usefulness of this expansion was made very clear in a paper authored by Ismail and Zhang, where it was used to solve the eigenvalue problem for the left inverse of the differential operator, on $L^{2}$ spaces with ultraspherical weights [20]. The consideration of the $q$-analogue of this diagonalization problem led the authors to extend Gegenbauer's formula to the $q$-case. This task required the introduction of a new $q$-analogue of the exponential, a two variable function denoted by $\mathcal{E}_{q}(x ; t)$ which became known in the literature as the curly $q$-exponential function, bearing the name from its

Date received: March 6, 2006. Date accepted: August 4, 2006. Communicated by Mourad Ismail. Online publication: September 17, 2007.

AMS classification: Primary 42C15, 44A20; Secondary 33C45, 33D45, 94A20.

Key words and phrases: Reproducing kernels, $q$-Fourier series, Orthogonal polynomials, Basic hypergeometric functions, Sampling theorems. 
notational convention. Ismail and Zhang's formula is

$$
\begin{aligned}
\mathcal{E}_{q}(x ; i t)= & \frac{t^{-v}(q ; q)_{\infty}}{\left(-q t^{2} ; q^{2}\right)_{\infty}\left(q^{v+1} ; q\right)_{\infty}} \\
& \times \sum_{k=0}^{\infty} i^{k} q^{k^{2} / 4} \frac{\left(1-q^{k+v}\right)}{\left(1-q^{\nu}\right)} J_{v+k}^{(2)}(2 t ; q) C_{k}\left(x ; q^{v} \mid q\right) .
\end{aligned}
$$

The functions involved in this formula will be defined in Section 4. Since its introduction, the function $\mathcal{E}_{q}$ was welcomed as a proper $q$-analogue of the exponential function, since it was suitable to provide a satisfactory $q$-analogue of the Fourier theory of integral transformations and series developments. This suitability was made concrete by Bustoz and Suslov in [5], where the authors introduced the subject of $q$-Fourier series. Some of the subsequent research activity has already been collected in a book [29]. Among recent developments not yet included in this book, we quote the orthogonality relations for sums of curly exponential functions [23], obtained using spectral methods, and the construction of a $q$-analogue of the Whittaker-Shannon-Kotel' nikov sampling theorem [19]. The designation "Quantum" has appear often in recent literature on $q$-analysis, as in the monographs [22] and [21]. This designation is very convenient, since $q$-special functions are intimately connected with representations of quantum groups [6].

An abstract formulation designed to capture the essential properties of $q$-Fourier-type systems was proposed in [15] and we proceed to describe it here. Let $\mu$ be a real measure on the real line and $\left\{p_{n}(x)\right\}$ be a complete orthonormal system in $L^{2}(\mu)$ and assume that $\left\{r_{n}(x)\right\}$ is a discrete orthonormal system whose orthogonality relation is

$$
\sum_{j=0}^{\infty} \rho\left(t_{j}\right) r_{n}\left(t_{j}\right) \overline{r_{m}\left(t_{j}\right)}=\delta_{m n}
$$

and with dual orthogonality

$$
\sum_{k=0}^{\infty} r_{k}\left(t_{n}\right) \overline{r_{k}\left(t_{m}\right)}=\frac{\delta_{m n}}{\rho\left(t_{n}\right)}
$$

Assume also that the system $\left\{r_{n}(x)\right\}$ is complete in $L^{2}\left(\sum \rho\left(t_{j}\right) \delta_{t_{j}}\right)$. Now define a sequence of functions $\left\{F_{n}(x)\right\}$ by

$$
F_{n}(x)=\sum_{k=0}^{\infty} r_{k}\left(t_{n}\right) p_{k}(x) u_{k},
$$

where $\left\{u_{k}\right\}$ is an arbitrary sequence of complex numbers in the unit circle. The following theorem is due to Ismail and comprises in an abstract form the fundamental fact behind the theory of basic analogs of Fourier series on a $q$-quadratic grid [5].

Theorem A [15]. The system $\left\{F_{n}(x)\right\}$ is orthogonal and complete in $L^{2}(\mu)$.

To give an idea of what is involved in this statement, we sketch Ismail's argument. Since, by (1.3), $r_{k}\left(t_{n}\right)$ are the Fourier coefficients of $F_{n}$ in the basis $\left\{u_{k} p_{k}\right\}$, the use of 
Parseval's formula gives

$$
\int F_{n}(x) \overline{F_{m}(x)} d \mu(x)=\sum_{k=0}^{\infty} r_{k}\left(t_{n}\right) \overline{r_{k}\left(t_{m}\right)}=\frac{\delta_{m n}}{\rho\left(x_{n}\right)}
$$

and the orthogonality relation is proved. To show the completeness, choose $f \in L^{2}(\mu)$ and assume $\int F_{n}(x) f(x) d \mu(x)=0$ for all $n$. Again Parseval's formula implies $\sum_{k=0}^{\infty} f_{k} \overline{r_{k}\left(t_{m}\right)}=0$ for all $m$, where $f_{k}$ are the Fourier coefficients of $f$ in the basis $\left\{p_{k}\right\}$. Now the completeness of $\left\{r_{k}\right\}$ implies $f_{k}=0$. Therefore $f=0$ almost everywhere in $L^{2}(\mu)$.

In [16] (see also Section 24.2 of the monograph [21]), Ismail posed the problem of studying the mapping properties of operators with kernels defined as above and conjectured that there was a reproducing kernel Hilbert space structure behind these operators. We will show that Ismail's conjecture is true. Our approach will reveal a reproducing kernel structure reminiscent of the well-known structure of the Paley-Wiener space of functions bandlimited to a real interval. However, even in the case when the system $\left\{F_{n}(x)\right\}$ is the set of the complex exponentials, we obtain results that, as far as our knowledge goes, seem to be new. When the system $\left\{F_{n}(x)\right\}$ is the set of basis functions of the $q$-Fourier series constructed with the function $\mathcal{E}_{q}(x ; i t)$, we will obtain results that complement the investigations done in [29] and [19]. In particular, it will be shown that a sampling theorem related to the one derived in [19] lives in a reproducing kernel Hilbert space and that the correspondent $q$-analogues of the Sinc function provide an orthogonal basis for that space.

Before outlining the paper we want to make clear that the techniques we are using already exist in some antecedent form. In particular, Section 2 contains ideas from Ismail's theory of generalized $q$-Fourier series and from Saitoh's theory of linear transformations in Hilbert space. However, their particular combination here leads to new conclusions and sheds new light in the emerging theory of $q$-Fourier series. It reveals an elementary structure underlying many systems involving several special functions simultaneously. The results in Section 2.2 and in Sections 3, 4 and 5 were never explicitly stated before.

This paper can be summarized as follows. The next section contains the description of the reproducing kernel structure behind the abstract setting of Theorem A. An integral transformation between two Hilbert spaces is defined in the context of Saitoh's theory of linear transformations, basis for both spaces are provided and the formula for the reproducing kernel of the image Hilbert space is deduced. In this context a sampling theorem appears, generalizing the one in [19]. The remaining sections consider three applications of these results, using specific systems of orthogonal polynomials as well as Bessel functions and their generalizations. The first application is associated to formula (1.1) and systems of complex exponentials. The reproducing kernel in this case is written in terms of Bessel functions. The second application is linked to (1.2) and to systems of curly $q$-exponentials, and we write the reproducing kernel as $\mathrm{a}_{2} \varphi_{2}$ basic hypergeometric function. These two examples explore the interplay between Lommel polynomials and Bessel functions and the corresponding relations between their $q$-analogues. In the last section we consider a construction of a general character, designed originally in the papers [20], [17] and [15]. It allows us to extend the interplay between Bessel functions and Lommel polynomials to a more general class of functions. Using this construction 
we will make a brief discussion about the application of our results to spaces weighted by Jacobi weights and their $q$-analogues.

\section{The Reproducing Kernel Structure}

Let $H$ be a class of complex valued functions, defined in a set $X \subset \mathbf{C}$, such that $H$ is a Hilbert space with the norm of $L^{2}(X, \mu)$. The function $k(s, x)$ is a reproducing kernel of $H$ if:

(i) $k(\cdot, x) \in H$ for every $x \in X$;

(ii) $f(x)=\langle f(\cdot), k(\cdot, x)\rangle$ for every $f \in H, x \in X$.

The space $H$ is said to be a reproducing kernel Hilbert space if it contains a reproducing kernel. From a structural point of view, the correct approach to the study of our problem is via Saitoh's theory of linear transforms of Hilbert space.

\subsection{Preliminaries on Saitoh's Theory of Linear Transformations in Hilbert Space}

This theory can be found in works by Saitoh [26], [27] and we proceed to give a brief account of the results that we are going to use. An account of the results quoted in this section can also be found in Higgins' recent survey [13].

For each $t$ belonging to a domain $D$, let $K_{t}$ belong to $H$ (a separable Hilbert space). Then,

$$
k(s, t)=\left\langle K_{s}, K_{t}\right\rangle_{H}
$$

is defined on $D \times D$ and is called the kernel function of the map $K_{t}$. Now consider the set of images of $H$ by the transformation

$$
(K g)(t)=\left\langle g, K_{t}\right\rangle_{H}=f(t)
$$

and denote this set of images by $R(K)$. The following theorem can be found in [26].

Theorem B. The kernel $k(x, t)$ determines uniquely a reproducing kernel Hilbert space for which it is the reproducing kernel. This reproducing kernel Hilbert space is precisely $R(K)$ and it can have no other reproducing kernel.

Now, suppose that $\left\{K_{t}\right\}(t \in D)$ is complete in $H$ so that $K$ is one-to-one. Then we have

$$
\|K g\|_{R(K)}=\|g\|_{H}
$$

The following theorem, due to Higgins [12], will be critical to the remainder. We will use only the following "orthogonal basis case", a special case of the theorem in [12]:

Theorem C. With the notations established earlier, we have: If there exists $\left\{t_{n}\right\}(n \in D)$ such that $\left\{K_{t_{n}}\right\}$ is an orthogonal basis, we then have the sampling expansion

$$
f(t)=\sum_{n \in D} f\left(t_{n}\right) \frac{k\left(t, t_{n}\right)}{k\left(t_{n}, t_{n}\right)}
$$


in $R$, pointwise over $D$, and uniform over any compact subset of $D$ for which $\left\|K_{t}\right\|$ is bounded.

\subsection{The Reproducing Kernel for q-Fourier-Type Systems}

In this section we will show the existence of a reproducing kernel structure behind the abstract setting of Theorem A. The results will follow from the study of the mapping properties of an integral transform whose kernel is obtained from the sequence of functions $\left\{r_{k}\right\}$ and $\left\{p_{k}\right\}$.

Theorem 1. There exists a kernel $K(x, t)$ satisfying the requirements of Saitoh's theory of linear transformations, such that $K\left(x, x_{n}\right)=\lambda_{n} F_{n}(x)$, where $\left\{F_{n}(x)\right\}$ is the orthogonal sequence of functions in Theorem $\mathrm{A}$ and $\left\{\lambda_{n}\right\}$ is a sequence of real numbers.

Proof. Our first technical problem comes from the fact that, when $\left\{r_{k}\right\}$ is a discrete system of orthogonal polynomials with a determinate moment problem, then $\left\{r_{k}(t)\right\} \in l^{2}$ if and only if $x$ is a mass point for the measure of orthogonality. For this reason the series

$$
\sum_{k=0}^{\infty} r_{k}(t) p_{k}(x) u_{k}
$$

would diverge if $t$ is not such a point (this is pointed out in Section 5 of [15]). Since we want our kernel to be defined for every $t$, we will assume the existence of an auxiliary system of functions $\left\{\mathcal{J}_{k}(x)\right\} \in l^{2}$ for every $t$ real, and such that every function $\mathcal{J}_{k}$ interpolates $r_{k}$ at the mass points $\left\{x_{n}\right\}$ in the sense that

$$
\mathcal{J}_{k}\left(x_{n}\right)=\lambda_{n} r_{k}\left(\frac{1}{x_{n}}\right)
$$

for every $k=0,1, \ldots$ and $n=0,1, \ldots$ and some constant $\lambda_{n}$ independent of $k$. Now we can use the functions $\mathcal{J}_{k}(t)$ to define a kernel $K(x, t)$ as

$$
K(x, t)=\sum_{k=0}^{\infty} \mathcal{J}_{k}(t) p_{k}(x) u_{k} .
$$

Such a kernel is well defined and belongs to $L^{2}(\mu)$, since it is a sum of basis functions of $L^{2}(\mu)$. From (1.3), (2.1) and (2.2) we have

$$
\begin{aligned}
K\left(x, x_{n}\right) & =\sum_{k=0}^{\infty} \mathcal{J}_{k}\left(x_{n}\right) p_{k}(x) u_{k} \\
& =\lambda_{n} \sum_{k=0}^{\infty} r_{k}\left(\frac{1}{x_{n}}\right) p_{k}(x) u_{k} \\
& =\lambda_{n} F_{n}(x) .
\end{aligned}
$$

Remark 1. Observe that Theorem 1 and Theorem A with $t_{n}=1 / x_{n}$ show that $K\left(x, x_{n}\right)$ is an orthogonal basis for the space $L^{2}(\mu)$. 
Remark 2. In the abstract formulation it may not be clear why the constant $\lambda_{n}$ must be present. Actually the construction would work without it, but for technical reasons that will become evident upon consideration of examples we prefer to use it. Otherwise, careful bookkeeping of the normalization constants would be required in the remaining sections.

Remark 3. It will be seen in the last section that a general constructive method is available in order to find the function $\mathcal{J}_{k}$ under very natural requirements on the polynomials $r_{k}$.

Now define an integral transformation $F$ by setting

$$
(F f)(t)=\int f(x) K(x, t) d \mu(x) .
$$

We will study this transform as a map whose domain is the Hilbert space $L^{2}(\mu)$. Endowing the range of $F$ with the inner product

$$
\langle F f, F g\rangle_{F\left(L^{2}(\mu)\right)}=\langle f, g\rangle_{L^{2}(\mu)},
$$

then $F\left(L^{2}(\mu)\right)$ becomes a Hilbert space isometrically isomorphic to $L^{2}(\mu)$ under the isomorphism $F$. Using Saitoh's theory with $D=\mathbf{R}, K_{t}=K(\cdot, t), H=L^{2}(\mu)$ and $R(K)=F\left(L^{2}(\mu)\right)$, we obtain at once:

Theorem 2. The transform $F$ is a Hilbert space isomorphism mapping the space $L^{2}(\mu)$ into $F\left(L^{2}(\mu)\right)$. The space $F\left(L^{2}(\mu)\right)$ is a Hilbert space with reproducing kernel given by

$$
k(t, s)=\int K(x, t) \overline{K(x, s)} d \mu(x)
$$

An interesting feature of this particular setting is that every function in $F\left(L^{2}(\mu)\right)$ has two different expansions: One is the sampling expansion naturally associated with the reproducing kernel structure, the other is the expansion in the basis $\mathcal{J}_{n}(x)$. It should be remarked that, in most of the previously known sampling expansions, these two expansions were the same. These expansion results are summarized in the next theorem.

Theorem 3. Every function $f$ of the form

$$
f(x)=\int u(t) K(t, x) d \mu(t)
$$

with $u \in L^{2}(\mu)$, admits an expansion

$$
f(t)=\sum_{n=0}^{\infty} a_{n} \mathcal{J}_{n}(t)
$$

where the coefficients $a_{k}$ are given by

$$
a_{n}=u_{n}\left\langle u, p_{n}(\cdot)\right\rangle_{L^{2}(\mu)}
$$


and a sampling expansion

$$
f(x)=\sum f\left(t_{n}\right) \frac{k\left(x, t_{n}\right)}{k\left(t_{n}, t_{n}\right)} .
$$

The sum in (2.7) converges absolutely. Furthermore, it converges uniformly in every set such that $\|K(\cdot, t)\|_{L^{2}(\mu)}$ is finite.

Proof. We already know by default that $\left\{p_{n}(x)\right\}$ is a basis for $L^{2}(\mu)$. It remains to prove that $\left\{\mathcal{J}_{n}(t)\right\}$ is a basis for $F\left(L^{2}(\mu)\right)$. Observe that

$$
\begin{aligned}
\left(F p_{n}\right)(t) & =\int p_{n}(x) K(x, t) d \mu(x) \\
& =\sum_{k=0}^{\infty} \mathcal{J}_{k}(t) u_{k} \int p_{n}(x) p_{k}(x) d \mu(x) \\
& =\mathcal{J}_{n}(t) u_{n} .
\end{aligned}
$$

Since $\left\{p_{n}(x)\right\}$ is a basis for $L^{2}(\mu)$ and $F$ is an isomorphism between $L^{2}(\mu)$ and $F\left(L^{2}(\mu)\right)$, then $\left\{\mathcal{J}_{n}(x)\right\}$ is a basis for $F\left(L^{2}(\mu)\right)$. To prove the last assertion of the theorem, observe that function $f$ defined by (2.5) belongs to $F\left(L^{2}(\mu)\right)$ and therefore can be expanded in the basis $\left\{u_{n} \mathcal{J}_{n}(t)\right\}$. The Fourier coefficients of this expansion are

$$
a_{n}=\left\langle f, u_{n} \mathcal{J}_{n}(\cdot)\right\rangle_{F\left(L^{2}(\mu)\right)}=\left\langle F u, F\left(p_{n}(\cdot)\right)\right\rangle_{F\left(L^{2}(\mu)\right)}=\left\langle u, p_{n}(\cdot)\right\rangle_{L^{2}(\mu)},
$$

where we have used (2.3) in the last identity. The sampling expansion follows from applying Theorem $\mathrm{C}$ to our setting and using Remark 1.

Remark 4. The construction of this section has never appeared before in the literature, but it is reminiscent of the reproducing kernel structure of the Paley-Wiener space. In the classical situations (see, e.g., [8] and [24] for an account of these constructions with several examples) generalizing this structure, there is an integral transform whose kernel is defined as

$$
K(x, t)=\sum S_{k}(t) e_{k}(x),
$$

where $e_{k}(x)$ is an orthogonal basis for the domain Hilbert space and $S_{k}(t)$ is a sequence of functions such that there exists a sequence $\left\{t_{n}\right\}$ satisfying the sampling property

$$
S_{k}\left(t_{n}\right)=a_{n} \delta_{n, k} .
$$

As an instance, take $S_{k}(t)=\sin \pi(t-k) / \pi(t-k)$ and $e_{k}(x)=e^{i k x}$. Then (2.8) is

$$
e^{i t x}=\sum_{k=-\infty}^{\infty} \frac{\sin \pi(t-k)}{\pi(t-k)} e^{i k x}
$$

and $K(x, t)$ is the kernel of the Fourier transform. The corresponding reproducing kernel Hilbert space is the Paley-Wiener space. The root of these ideas is in Hardy's groundbreaking paper [11]. For an application of this classical set-up to Jackson $q$-integral 
transforms and the third Jackson $q$-Bessel function, see [1]. In our construction we made a modification of this classical setting: Instead of the sequence of functions $S_{k}$, with the sampling property (2.9), we considered a sequence of functions $\left\{\mathcal{J}_{k}\right\}$, interpolating an orthogonal system $\left\{r_{k}\right\}$ in the sense of (2.1). And we have seen that the essential properties of classical reproducing kernel settings are kept. However, this modification allows us to recognize a class of reproducing kernel Hilbert spaces that were obscured until now. This will become clear in the next section.

\section{The Fourier System with Ultraspherical Weights}

The $n$th ultraspherical (or Gegenbauer) polynomial of order $v$ is denoted by $C_{n}^{v}(x)$. These polynomials satisfy the orthogonality relation

$$
\int_{-1}^{1} C_{n}^{v}(x) C_{m}^{v}(x)\left(1-x^{2}\right)^{\nu-1 / 2} d x=\frac{(2 v)_{n} \sqrt{\pi} \Gamma\left(v+\frac{1}{2}\right)}{n !(v+n) \Gamma(v)} \delta_{m, n},
$$

and form a complete sequence in the Hilbert space $L^{2}\left[(-1,1),\left(1-x^{2}\right)^{\nu-1 / 2}\right]$. For typographical convenience we will introduce the following notation for this Hilbert space:

$$
H^{v}=L^{2}\left[(-1,1),\left(1-x^{2}\right)^{v-1 / 2}\right] .
$$

The Bessel function of order $v, J_{v}(x)$, is defined by the power series expansion

$$
J_{v}(z)=\sum_{n=0}^{\infty} \frac{(-1)^{n}}{n ! \Gamma(v+n+1)}\left(\frac{z}{2}\right)^{v+2 n} .
$$

The $n$th Lommel polynomial of order $v$, denoted by $h_{n, v}(x)$, is related to the Bessel functions by the relation

$$
J_{v+k}(x)=h_{k, v}\left(\frac{1}{x}\right) J_{v}(x)-h_{k-1, v-1}\left(\frac{1}{x}\right) J_{v-1}(x) .
$$

The Lommel polynomials satisfy the discrete orthogonality relation

$$
\sum_{k=0}^{\infty} \frac{1}{\left(j_{v, k}\right)^{2}} h_{n, v+1}\left( \pm \frac{1}{j_{v, k}}\right) h_{m, v+1}\left( \pm \frac{1}{j_{v, k}}\right)=\frac{\delta_{n m}}{2(v+n+1)}
$$

and the dual orthogonality

$$
\sum_{k=0}^{\infty} 2(v+n+1) h_{k, v+1}\left( \pm \frac{1}{j_{v, n}}\right) h_{k, v+1}\left( \pm \frac{1}{j_{v, m}}\right)=\left(j_{v, k}\right)^{2} \delta_{n m}
$$

They form a complete orthogonal system in the $l^{2}$-space weighted by the discrete measure with respect to which they are orthogonal. We will use these two complete orthogonal systems in our first illustration of the general results. Set

$$
p_{k}(x)=\sqrt{\frac{k !(v+k)}{(2 v)_{k}}} C_{k}^{v}(x)
$$


and

$$
r_{k}(t)=\sqrt{2(v+n)} h_{k, v-1}(t)
$$

Consider also

$$
\mathcal{J}_{k}(t)=\sqrt{2(v+n)} J_{v+k}(t) .
$$

Denote by $j_{v, k}$ the $k$ th zero of the Bessel function of order $v$. Substituting $x=j_{v, n}$ in (3.2), the following interpolating property is obtained:

$$
h_{k, v-1}\left(\frac{1}{j_{v, n}}\right)=-\frac{J_{v+k}\left(j_{v, n}\right)}{J_{v-1}\left(j_{v, n}\right)} .
$$

The interpolating property (3.3) will play the role of (2.1) with $\lambda_{n}=-1 / J_{v-1}\left(j_{v, n}\right)$. Consider also the sequence of complex numbers $\left\{u_{n}\right\}$ defined as

$$
u_{k}=i^{k}
$$

and set

$$
K^{v}(x, t)=\sqrt{2} \sum_{k=0}^{\infty} i^{k}(v+k) \sqrt{\frac{2 k !}{(2 v)_{k}}} J_{v+k}(t) C_{k}^{v}(x) .
$$

Now, Theorem 1 tells us that $K^{v}\left(x, j_{v, n}\right)$ is an orthogonal basis of the space $H^{v}$. Moreover, formula (1.1) implies

$$
\sqrt{\frac{\pi}{2 t}} K^{1 / 2}(x, t)=e^{i x t}
$$

and, therefore, we can think of $\Gamma(v)(1 / 2 t)^{v} K^{v}(x, t)$ as a one-parameter generalization of the complex exponential kernel that may be worth further study. In the special case $v=\frac{1}{2}$ we recover the well-known orthogonality and completeness of the complex exponentials $\left\{e^{i \pi n x}\right\}$ in $L^{2}(-1,1)$.

The transformation $F$ is defined, for every $f \in H^{\nu}$, as

$$
(F f)(t)=\int_{-1}^{1} f(x) K^{v}(x, t)\left(1-x^{2}\right)^{v-1 / 2} d x
$$

and Theorem 2 gives that the reproducing kernel of $F\left(H^{v}\right)$ is

$$
k^{\nu}(t, s)=\int_{-1}^{1} K^{\nu}(x, t) \overline{K^{\nu}(x, s)}\left(1-x^{2}\right)^{\nu-1 / 2} d x .
$$

When $v=\frac{1}{2}$ this becomes

$$
k^{1 / 2}(t, s)=\frac{\pi \sqrt{t s}}{2} \frac{\sin (t-s)}{(t-s)} .
$$

Since $\left\{\sqrt{\left[k !(v+k) /(2 v)_{k}\right]} C_{n}^{v}(t)\right\}$ forms a basis of the space $H^{v}$, then also $\left\{i^{n} \sqrt{2(v+n)} J_{v+n}(x)\right\}=F\left\{\sqrt{\left[k !(v+k) /(2 v)_{k}\right]} C_{n}^{v}(t)\right\}$ is a basis of the space $F\left(H^{v}\right)$. In this situation Theorem 3 reads: 
Theorem 4. Let $f$ be a function of the form

$$
f(t)=\int_{-1}^{1} u(x) K^{v}(x, t)\left(1-x^{2}\right)^{\nu-1 / 2} d x
$$

where $u \in H^{\nu}$. Then $f$ can be written as

$$
f(t)=\sum_{n=0}^{\infty} a_{n} J_{v+n}(t)
$$

with the coefficients $a_{n}$ given by

$$
a_{n}=i^{n}(v+n) \sqrt{\frac{2 n !}{(2 v)_{n}}} \int_{-1}^{1} u(x) C_{n}^{v}(x)\left(1-x^{2}\right)^{\nu-1 / 2} d x
$$

and also as the sampling formula

$$
f(t)=\sum_{n=0}^{\infty} f\left(j_{v, n}\right) \frac{k^{v}\left(t, j_{v, n}\right)}{k^{v}\left(j_{v, n}, j_{v, n}\right)}
$$

Remark 5. Expansions of the type (3.5) are known as Neumann series of Bessel functions (see Chapter 16 of [25]).

Remark 6. When $v=\frac{1}{2}$ the above sampling theorem states that every function of the form

$$
f(t)=\left(\frac{t}{2}\right)^{-1 / 2} \int_{-1}^{1} u(x) e^{i x t} d x
$$

with $u \in L^{2}[(-1,1)]$, can be represented as

$$
f(t)=\sum_{n=0}^{\infty} f(2 \pi n) \sqrt{\frac{t}{2 \pi n}} \frac{\sin (t-2 \pi n)}{(t-2 \pi n)} .
$$

\section{The $q$-Fourier System with $q$-Ultraspherical Weights}

We proceed to describe the $q$-analogue of the previous situation. Choose a number $q$ such that $0<q<1$. The now classical notational conventions from [9] and [21] for $q$-infinite products and basic hypergeometric series will be used often.

The $q$-exponential function that we talked about in the introduction is defined in terms of basic hypergeometric series as

$$
\mathcal{E}_{q}(x ; t)=\frac{\left(-t ; q^{1 / 2}\right)_{\infty}}{\left(q t^{2} ; q^{2}\right)_{\infty}}{ }_{2 \varphi_{1}}\left(\begin{array}{c}
q^{1 / 4} e^{i \theta}, q^{1 / 4} e^{-i \theta} \\
q^{1 / 2}
\end{array} \mid q^{1 / 2},-t\right)
$$


where $x=\cos \theta$. The continuous $q$-ultraspherical polynomials of order $v$ are denoted by $C_{n}^{v}\left(x ; q^{v} \mid q\right)$ and satisfy the orthogonality

$$
\begin{aligned}
\int_{-1}^{1} C_{n}^{v}\left(x ; q^{v} \mid q\right) C_{m}^{v}\left(x ; q^{v} \mid q\right) w\left(x ; q^{v} \mid q\right) d x \\
=\frac{\left(2 \pi q^{v}, q^{v+1} ; q\right)_{\infty}}{\left(q, q^{2 \nu} ; q\right)_{\infty}} \frac{\left(1-q^{\nu}\right)\left(q^{2 v} ; q\right)_{n}}{\left(1-q^{n+v}\right)(q ; q)_{n}} \delta_{m, n}
\end{aligned}
$$

where the weight function $w(x ; \beta \mid q)$ is

$$
w(\cos \theta ; \beta \mid q)=\frac{\left(e^{2 i \theta}, e^{-2 i \theta} ; q\right)_{\infty}}{\left.\sin \theta\left(\beta e^{2 i \theta}, \beta e^{-2 i \theta} ; q\right)\right)_{\infty}} \quad(0<\theta<\pi) .
$$

The polynomials $\left\{C_{n}^{v}\left(x ; q^{v} \mid q\right)\right\}$ form a basis of the Hilbert space $H_{q}^{v}$ defined as

$$
H_{q}^{v}=L^{2}\left[(-1,1), w\left(x ; q^{v} \mid q\right)\right] .
$$

The second Jackson $q$-Bessel function of order $v$ is defined by the power series

$$
J_{v}^{(2)}(x ; q)=\frac{\left(q^{v+1} ; q\right)_{\infty}}{(q ; q)_{\infty}} \sum_{k=0}^{\infty}(-1)^{n} \frac{(x / 2)^{v+2 n}}{(q ; q)_{n}\left(q^{\nu+1} ; q\right)_{n}} q^{n(v+n)} .
$$

Since this is the only $q$-Bessel function to be used in the text, we will drop the superscript for brevity of notation and write $J_{v}(x ; q)=J_{v}^{(2)}(x ; q)$. The $q$-Lommel polynomials associated to the Jackson $q$-Bessel function of order $v$ are denoted by $h_{n, v-1}(x ; q)$. These polynomials were defined in [14] by means of the relation

$$
q^{n v+n(n-1) / 2} J_{v+n}(x ; q)=h_{n, v}\left(\frac{1}{x} ; q\right) J_{v}(x ; q)-h_{n-1, v-1}\left(\frac{1}{x} ; q\right) J_{v-1}(x ; q) .
$$

The $q$-Lommel polynomials satisfy the orthogonality relation

$$
\sum_{k=1}^{\infty} \frac{A_{k}(v+1)}{\left(j_{v, n}(q)\right)^{2}} h_{n, v+1}\left( \pm \frac{1}{j_{v, n}(q)} ; q\right) h_{m, v+1}\left( \pm \frac{1}{j_{v, n}(q)} ; q\right)=\frac{q^{n v+n(n+1) / 2}}{1-q^{n+v+1}} \delta_{n m}
$$

and the dual orthogonality

$$
\sum_{k=1}^{\infty} \frac{\left(1-q^{n+v+1}\right)}{q^{n v+n(n+1) / 2}} h_{n, v+1}\left( \pm \frac{1}{j_{v, n}(q)} ; q\right) h_{m, v+1}\left( \pm \frac{1}{j_{v, n}(q)} ; q\right)=\frac{\left(j_{v, n}(q)\right)^{2}}{A_{k}(v+1)} \delta_{n m}
$$

Consider

$$
\begin{aligned}
& p_{k}(x)=\sqrt{\frac{\left(1-q^{k+v}\right)(q ; q)_{k}}{\left(1-q^{v}\right)\left(q^{2 v} ; q\right)_{k}}} C_{k}\left(x ; q^{v} \mid q\right), \\
& r_{k}(t)=\sqrt{\frac{\left(1-q^{k+v}\right)}{\left(1-q^{\nu}\right)}} q^{-k v / 2-k(k-1) / 4} h_{k, v}(2 t ; q),
\end{aligned}
$$


and

$$
\mathcal{J}_{k}(t)=\sqrt{\frac{\left(1-q^{k+v}\right)}{\left(1-q^{v}\right)}} q^{k v / 2+k(k-1) / 4} J_{v+k}(2 t ; q) .
$$

The parameters $u_{k}$ will be given by

$$
u_{k}=i^{k} .
$$

Denote by $j_{v, k}(q)$ the $k$ th zero of $J_{v}(x ; q)$. Setting $t=j_{v, k}(q)$ in (4.1) we have the interpolating property

$$
h_{k, v-1}\left(\frac{1}{j_{v, n}(q)} ; q\right)=-q^{k v+k(k-1) / 2} \frac{J_{v+k}\left(j_{v, n}(q) ; q\right)}{J_{v-1}\left(j_{v, n}(q) ; q\right)} .
$$

This means that in (2.1) we must take $\lambda_{n}=-1 / J_{v-1}\left(j_{v, n}(q) ; q\right)$. In this context, the kernel $K(x, t)$ is given as

$$
K_{q}^{v}(x, t)=\sum_{k=0}^{\infty} i^{k} q^{k(2 v+k-1) / 4} \frac{\left(1-q^{k+v}\right)}{\left(1-q^{v}\right)} \sqrt{\frac{(q ; q)_{k}}{\left(q^{2 v} ; q\right)_{k}}} J_{v+k}(2 t ; q) C_{k}\left(x ; q^{v} \mid q\right)
$$

and the use of (1.2) gives, when $v=\frac{1}{2}$,

$$
K_{q}^{1 / 2}(x, t)=\frac{\left(-q t^{2} ; q^{2}\right)_{\infty}\left(q^{v+1} ; q\right)_{\infty}}{(q ; q)_{\infty}} t^{\nu} \mathcal{E}_{q}(x ; i t) .
$$

The basis functions of the domain space are

$$
F_{n}(x)=K_{q}^{v}\left(x, j_{v, k}(q)\right) .
$$

When $v=\frac{1}{2}$ this gives that $\left\{\mathcal{E}_{q}\left(x ; i j_{1 / 2, n-1}(q)\right)\right\}$ is orthogonal and complete in $H_{q}^{v}$. This is the case of the $q$-Fourier series studied in [29]. Now define the transform

$$
\left(F_{q}^{v} f\right)(t)=\int_{-1}^{1} f(x) K_{q}^{v}(x, t) w\left(x ; q^{v} \mid q\right) d x
$$

for every $f \in H_{q}^{v}$. We have

$$
i^{k} \sqrt{\frac{\left(1-q^{k+v}\right)}{\left(1-q^{\nu}\right)}} q^{k v / 2+k(k-1) / 4} J_{v+k}(2 t ; q)=F_{q}^{v}\left(\sqrt{\frac{\left(1-q^{k+v}\right)(q ; q)_{k}}{\left(1-q^{\nu}\right)\left(q^{2 \nu} ; q\right)_{k}}} C_{k}\left(x ; q^{v} \mid q\right)\right)
$$

and $\left\{J_{v+n}(t ; q)\right\}_{n=0}^{\infty}$ is a basis of the space $F_{q}^{v}\left(H_{q}^{v}\right)$. We can also state a $q$-Neumann expansion theorem in $q$-Bessel functions.

Theorem 5. Let $f$ be a function of the form

$$
f(t)=\int_{-1}^{1} u(x) K_{q}^{v}(x, t) w\left(x ; q^{v} \mid q\right) d x
$$


where $u \in H_{q}^{v}$. Then $f$ can be written as

$$
f(t)=\sum_{n=0}^{\infty} a_{n} J_{v+n}(t ; q)
$$

with the coefficients $a_{n}$ given by

$$
a_{n}=i^{n} \frac{\left(1-q^{k+v}\right)}{\left(1-q^{v}\right)} \sqrt{\frac{(q ; q)_{k}}{\left(q^{2 \nu} ; q\right)_{k}}} \int_{-1}^{1} u(x) C_{n}\left(x ; q^{v} \mid q\right) w\left(x ; q^{v} \mid q\right) d x .
$$

Once more we know by Theorem 2 that the space $F_{q}^{v}\left(H_{q}^{v}\right)$ is a space with reproducing kernel $k_{q}^{v}(t, s)$, given by

$$
k_{q}^{v}(t, s)=\int_{-1}^{1} K_{q}^{v}(x, t) \overline{K_{q}^{v}(x, s)} w\left(x ; q^{v} \mid q\right) d x .
$$

When $v=\frac{1}{2}$ we can compute the reproducing kernel in an explicit form using the following integral, evaluated in [18]:

$$
\begin{aligned}
\int_{0}^{\pi} & \mathcal{E}_{q}(\cos \theta ; \alpha) \mathcal{E}_{q}(\cos \theta ; \beta) \frac{\left(e^{2 i \theta}, e^{-2 i \theta} ; q\right)_{\infty}}{\left(\gamma e^{2 i \theta}, \gamma e^{-2 i \theta} ; q\right)_{\infty}} d \theta \\
\quad= & \frac{2 \pi\left(\gamma, q \gamma,-\alpha \beta q^{1 / 2} ; q\right)_{\infty}}{\left(q, \gamma^{2} ; q\right)_{\infty}\left(q \alpha^{2}, q \beta^{2} ; q^{2}\right)_{\infty}} 2 \varphi_{2}\left(\begin{array}{c}
-q^{1 / 2} \alpha / \beta,-q^{1 / 2} \beta / \alpha \\
q \gamma,-\alpha \beta \gamma q^{1 / 2}
\end{array} \mid q,-\alpha \beta \gamma q^{1 / 2}\right)
\end{aligned}
$$

Theorem 6. The space $F_{q}^{1 / 2}\left(H_{q}^{1 / 2}\right)$ is a space with reproducing kernel $k_{q}^{1 / 2}(t, s)$, given by

$$
\begin{aligned}
k_{q}^{\nu}(t, s)= & 2 \pi\left[\frac{\left(q^{3 / 2} ; q\right)_{\infty}^{3 / 2}}{(q ; q)_{\infty}^{2}}\right]^{2} \\
& \times\left(q^{1 / 2},-t s q^{1 / 2} ; q\right)_{\infty}(t s)^{1 / 2}{ }_{2} \varphi_{2}\left(\begin{array}{c}
-q^{1 / 2} t / s,-q^{1 / 2} s / t \\
q^{3 / 2},-t s q
\end{array} \mid q,-t s q\right) .
\end{aligned}
$$

Proof. Applying Theorem 2 and (4.3) we know that $k_{q}^{v}(t, s)$ is given by

$$
\begin{aligned}
k_{q}^{v}(t, s)= & {\left[\frac{\left(q^{v+1} ; q\right)_{\infty}}{(q ; q)_{\infty}}\right]^{2}\left(-q t^{2},-q s^{2} ; q^{2}\right)_{\infty}(t s)^{1 / 2} } \\
& \times \int_{-1}^{1} \mathcal{E}_{q}(x ; i t) \mathcal{E}_{q}(x ;-i s) w\left(x ; q^{v} \mid q\right) d x .
\end{aligned}
$$

Make the substitutions $x=\cos \theta$, it $=\alpha$, is $=\beta$, and $q^{\nu}=\gamma$ in (4.6). Then (4.7) follows.

As in the preceding sections we can formulate a sampling theorem. 
Theorem 7. Every function of the form (4.5) admits the expansion

$$
f(x)=\sum_{k=0}^{\infty} f\left(t_{k}\right) \frac{k_{q}^{v}\left(x, t_{k}\right)}{k_{q}^{v}\left(t_{k}, t_{k}\right)}
$$

where $t_{k}=j_{v, k}(q) / 2$.

Remark 7. The functions $k_{q}^{v}\left(x, t_{k}\right) / k_{q}^{v}\left(t_{k}, t_{k}\right)$ are related to the functions $\operatorname{Sinc}_{q}(t, k)$ from [19]. In [19] it is shown that there is a sampling theorem associated to the $\operatorname{Sinc}_{q}(t, k)$ functions that can be written as an interpolating formula of the Lagrange type. The above discussion shows what is the sampling theorem associated to the reproducing kernel Hilbert space and its relation to the one obtained in [19].

Remark 8. By the proof of Theorem B (see [13]), the functions $k_{q}^{v}\left(x, t_{k}\right) / k_{q}^{v}\left(t_{k}, t_{k}\right)$ are orthogonal in the space $F_{q}^{1 / 2}\left(H_{q}^{1 / 2}\right)$. This result is a $q$-analogue of the important fact, proved by Hardy in [11], that the functions $\sin \pi(t-k) / \pi(t-k)$ are orthogonal in the classical Paley-Wiener space.

Remark 9. Important information concerning the zeros of the second Jackson $q$-Bessel function, that appear as sampling nodes in the expansion (4.7), was obtained very recently by Walter Hayman in [10] using a method due to Bergweiler and Hayman [4]: the asymptotic expansion

$$
j_{v, k}^{2}(q)=4 q^{1-2 n-v}\left\{1+\sum_{\nu=1}^{n} b_{\nu} q^{k v}+O\left|q^{(n+1) k}\right|\right\}
$$

as $k \rightarrow \infty$, with the constants $b_{v}$ depending on $a$ and $q$, holds. Therefore, for large $k$, the sampling nodes are exponentially separated in a similar way to that which was observed in [1] and [2]. In the case where $v= \pm \frac{1}{2}$, the zeros were studied by Suslov [28].

\section{A Generalization}

We begin this last section describing a formal approach generalizing the situations studied in the previous two sections. This formal approach was initiated in [20] and [17] with the purpose of finding functions to play the role of the Lommel polynomials in more general situations, and was studied further in [15]. In the context studied in this paper, it will be of particular relevance, since it gives a constructive method to find the functions $\mathcal{J}_{k}$ satisfying (2.1). Let $\left\{f_{n, v}\right\}$ be a sequence of polynomials defined recursively by $f_{0, v}(x)=1, f_{1, v}(x)=x B_{v}$ and

$$
f_{n+1, v}(x)=\left[x B_{n+v}\right] f_{n, v}(x)-C_{n+v-1} f_{n-1, v}(x) .
$$

Assuming the positivity condition $B_{n+v} B_{n+v+1} C_{n+v}>0(n \geq 0)$ and the convergence of the series $\sum_{n=0}^{\infty} C_{n+v} / B_{n+v} B_{n+v+1}$, it can be shown, using facts from the general theory of orthogonal polynomials, that the polynomials $f_{n, v}$ are orthogonal with respect 
to a compact supported discrete measure and that the support points of this measure are $1 / x_{n, v}$, where the $x_{n, v}$ are the zeros of an entire function $\mathcal{J}$ satisfying

$$
C_{v} \cdots C_{v+n-1} \mathcal{J}(x ; v+n)=\mathcal{J}(x ; v) f_{n, v}\left(\frac{1}{x}\right)-\mathcal{J}(x ; v-1) f_{n-1, v+1}\left(\frac{1}{x}\right)
$$

The dual orthogonality relation of the polynomials $f_{n, v}(x)$ is

$$
\sum_{n=0}^{\infty} \frac{B_{v+1}}{2 \lambda_{n}(v+1)} f_{n, v+1}\left(\frac{1}{x_{v, k}}\right) f_{n, v+1}\left(\frac{1}{x_{v, j}}\right)=\frac{x_{v, j}^{2}}{A_{j}(v+1)} \delta_{j, k}
$$

for some constants $A_{j}(v+1)$ and $\lambda_{n}(v+1)$. (For the evaluation of these constants, as well as other parts of the argument missed in this brief sketch, we recommend the reading of Section 4 of [15].) From (5.1) and the above analysis we can obtain the interpolation property

$$
\mathcal{J}\left(x_{n, v} ; v+k\right)=\frac{-\mathcal{J}\left(x_{n, v} ; v-1\right)}{C_{v} \cdots C_{v+n-1}} f_{k-1, v+1}\left(\frac{1}{x_{n, v}}\right) .
$$

Therefore, in the language of the second section we can set

$$
\begin{aligned}
\mathcal{J}_{k}(t) & =\sqrt{\lambda_{k}(v)} \frac{B_{k+v}}{B_{v}} \mathcal{J}(t, k), \\
r_{k}(t) & =\sqrt{\lambda_{k}(v)} \frac{B_{k+v}}{B_{v}} f_{n, v}(t), \\
\lambda_{n} & =\frac{-\mathcal{J}\left(x_{n, v} ; v-1\right)}{C_{v} \cdots C_{v+n-1}},
\end{aligned}
$$

and define the kernel

$$
K(x, t)=\sum_{k=0}^{\infty} u_{k} \sqrt{\lambda_{k}(v)} \frac{B_{k+v}}{B_{v}} \mathcal{J}(t, k) p_{k}(x),
$$

where $\left|u_{k}\right|=1$ and $\left\{p_{n}(x)\right\}$ is an arbitrary complete orthonormal system in $L^{2}(\mu)$. As before, the kernel $K(x, t)$ can be used to define an integral transformation between two Hilbert spaces. We could now apply the machinery of Section 2 and provide a reproducing kernel structure and a sampling theorem by means of an integral transform with the above kernel. However, no simplification would occur on the absence of proper addition formulas for the kernel $K(x, t)$. Choosing families of orthogonal polynomials $f_{n, v}(t)$ and $p_{n}(x)$ in a way that such addition formulas exist is the topic of the second problem in [16].

Operators weighted by the Jacobi weight function $(1-x)^{\alpha}(1+x)^{\beta}$ on the interval $[-1,1]$ can be studied by using the Jacobi polynomials and the Wimp polynomials in a similar fashion to that which was done in Section 4 of [20]. Reasoning as before, we can use the resulting formulas to generalize the results in the third section to Fourier systems with Jacobi weights, although no major simplification seems to occur. Results very similar to those of Section 3 would follow, with an extra parameter; expansions in 
series of ${ }_{1} F_{1}$ replace the Neumann expansions and sampling theorems with sampling points located at the zeros of these ${ }_{1} F_{1}$ can also be derived.

In Section 6 of [17], formula (6.13) is a generalization of (1.2). This formula involves continuous $q$-analogues of the Jacobi polynomials defined via the Askey-Wilson polynomials [3] and a $q$-exponential function with an extra variable. This more general and complicated situation should be studied in a future work once more summation formulas are known. Also, following Ismail's suggestion in Problem 24.2.1 of [21] may lead to the discovery of other examples of structures fitting to the setting described in this paper.

Acknowledgments. I thank Hans Feichtinger for his kind hospitality during my stay at NuHag, University of Vienna, where part of this work was done. I'm also indebted to Jose Carlos Petronilho and Fethi Bouzeffour for important discussions on earlier versions of the manuscript. Partial financial assistance was provided by FCT grant SFRH/BPD/26078/2005 and CMUC.

\section{References}

[1] L. D. ABREU (2005): A q-sampling theorem related to the q-Hankel transform. Proc. Amer. Math. Soc., 133:1197-1203.

[2] L. D. ABREU, J. Bustoz, J. L. CARDOSO (2003): The roots of the third Jackson q-Bessel function. Int. J. Math. Math. Sci., 67:4241-4248.

[3] R. ASKEY, J. WILSON (1985): Some basic hypergeometric orthogonal polynomials that generalize Jacobi polynomials. Mem. Amer. Math. Soc., 54(319).

[4] W. BERGWEILER, W. K. HAYMAN (2003): Zeros of solutions of a functional equation. Comput. Methods Funct. Theory, 3(1-2):55-78.

[5] J. Bustoz, S. K. SUSLOV (1998): Basic analog of Fourier series on a q-quadratic grid. Meth. Appl. Anal., 5(1):1-38.

[6] V. CharI, A. Pressley (1994): A Guide to Quantum Groups. Cambridge: Cambridge University Press.

[7] A. ERdelyi, W. Magnus, F. Oberhettinger, F. TRicomi (1953): Higher Transcendental Functions, Vol. 2. New York: McGraw-Hill.

[8] A. G. GARCIA (2000): Orthogonal sampling formulas: A unified approach. SIAM Rev., 42(3):499-512.

[9] G. GASPER, M. RAHMAN (1990): Basic Hypergeometric Series. Cambridge: Cambridge University Press.

[10] W. K. Hayman (2005): On the zeros of a q-Bessel function. In: Complex Analysis and Dynamical Systems, Vol. II, pp. 205-216. Contemp. Math., Vol. 382. Providence, RI: Amer. Math. Soc.

[11] G. H. HARDY (1941): Notes on special systems of orthogonal functions. IV. The orthogonal functions of Whittaker's cardinal series. Proc. Cambridge Philos. Soc., 37:331-348.

[12] J. R. HIGGINS (2001): A sampling principle associated with Saitoh's fundamental theory of linear transformations. In: Analytic Extension Formulas and Their Applications (Fukuoka, 1999/Kyoto, 2000), pp. 73-86. Int. Soc. Anal. Appl. Comput., Vol. 9. Dordrecht: Kluwer Academic.

[13] J. R. HIGGINS (2006): Integral transforms and sampling theorems. Integral Transforms Spec. Funct., 17(1):45-52.

[14] M. E. H. IsmaIL (1982): The zeros of basic Bessel functions, the functions $J_{v+a x}(x)$, and associated orthogonal polynomials. J. Math. Anal. Appl., 86:1-19.

[15] M. E. H. IsmaIL (2001): Orthogonality and completeness of q-Fourier-type systems. Z. Anal. Anwendungen, 20(3):761-775

[16] M. E. H. IsmaIL (2005): Problem 5. Orthogonality and completeness. In: Open Problems. J. Comput. Appl. Math., 178(1-2).

[17] M. E. H. ISMAIL, M. RAHMAN, R. ZHANG (1996): Diagonalization of certain integral operators. II. J. Comput. Appl. Math., 68(1-2):163-196. 
[18] M. E. H. ISMAIL, D. W. STANTON (2000): Addition theorems for the $q$-exponential function. In: $q$-Series from a Contemporary Perspective (South Hadley, MA, 1998), pp. 235-245. Contemp. Math., Vol. 254. Providence, RI: Amer. Math. Soc.

[19] M. E. H. ISMAIL, A. I. ZAYED (2003): A q-analogue of the Whittaker-Shannon-Kotelnikov sampling theorem. Proc. Amer. Math. Soc., 131(12):3711-3719

[20] M. E. H. ISMAIL, R. ZHANG (1994): Diagonalization of certain integral operators. Adv. Math., 109(1):133.

[21] M. E. H. IsmaIL (2005): Classical and Quantum Orthogonal Polynomials in One Variable. In: Encyclopedia of Mathematics and its Applications. Cambridge: Cambridge University Press.

[22] V. KAC, P. ChEung (2002): Quantum Calculus. New York: Springer-Verlag.

[23] E. KoELINK (2003): One-parameter orthogonality relations for basic hypergeometric series. Indag. Math. (N.S.), 14(3-4):423-443.

[24] M. Z NASHED, G. G. WALTER (1991): General sampling theorems for functions in reproducing kernel Hilbert spaces. Math. Control Signals Systems, 4(4):363-390.

[25] G. N WATSON (1995): A Treatise on the Theory of Bessel Functions. Reprint of the second 1944 edition. Cambridge Mathematical Library. Cambridge: Cambridge University Press.

[26] S. SAITOH (1988): Integral Transforms, Reproducing Kernels and Their Applications. Harlow: Longman.

[27] S. SAITOH (1995): One approach to some general integral transforms and its applications. Integral Transforms Spec. Funct., 3(1):49-84.

[28] S. K. SUSLOV (2003): Asymptotics of zeros of basic sine and cosine functions. J. Approx. Theory, 121(2):292-335.

[29] S. K. SUSLOV (2003): An Introduction to Basic Fourier Series. With a foreword by Mizan Rahman. Developments in Mathematics, Vol. 9. Dordrecht: Kluwer Academic.

\author{
L. D. Abreu \\ Department of Mathematics \\ University of Coimbra \\ Apartado 3008 \\ 3001-454 Coimbra \\ Portugal \\ daniel@mat.uc.pt \\ and \\ NUHAG \\ Faculty of Mathematics \\ University of Vienna \\ Nordbergstrasse 15 \\ A-1090 Wien \\ Austria
}

